\title{
VILLOUS LYMPHOCYTES IN BLOOD AND BONE MARROW IN SOME FORMS OF B-CELL LYMPHOID MALIGNANCIES
}

\author{
Alyona Polishchuk \\ Department of Oncohematology $y^{1}$ \\ alyona.p270189@gmail.com \\ Michael Zavelevich \\ Department of Oncohematology \\ mzavelevych@yahoo.com \\ Daniil Gluzman \\ Department of Oncohematology \\ vals@onconet.kiev.ua
}

${ }^{1}$ R. E. Kavetsky Institute of Experimental Pathology, Oncology and Radiobiology, National Academy of Sciences of Ukraine

45 Vasylkivska str., Kyiv, Ukraine, 03022

\begin{abstract}
The cytological and immunocytochemical features of the lymphocytes with villous morphology in peripheral blood and bone marrow in some B-lymphoproliferative disorders were studied. The diagnosis of hairy cell leukemia, a hairy cell leukemia variant, splenic marginal zone lymphoma and splenic diffuse red pulp small B-cell lymphoma was ascertained in accordance with the new revision of the WHO classification (2016). The neoplastic cells of hairy cell leukemia were determined by the presence of high tartrate resistant acid phosphatase (TRAP) activity. Cell surface expression of CD19, CD20 and CD21 antigens was detected. Also, the expression of CD25, CD103 and CD200, and in some cases cyclin D1, was found out. CD5, CD10 and CD23 were not detected. The immunophenotype of cells in splenic marginal zone lymphoma with villous processes also corresponded to the mature $\mathrm{B}$ cells. The expression of CD19, CD20 and CD21 was observed in all cases, CD11c - in $50 \%$ of patients, CD25 or CD5 - in $10 \%$ of patients. In $80 \%$ of patients, the pathologic cells did not show TRAP activity. In the bone marrow and peripheral blood cells of patients with diffuse red pulp lymphoma, TRAP activity was not detected. An immunophenotype in the hairy cell leukemia variant was different from those of classic $\mathrm{HCL}\left(\mathrm{CD} 19^{+} \mathrm{CD} 20^{+} \mathrm{CD} 22^{+} \mathrm{CD} 103^{+} \mathrm{CD} 11 \mathrm{c}^{+} \mathrm{CD} 5^{-} \mathrm{CD} 10^{-} \mathrm{CD} 23^{-}\right)$. Characterized immunophenotypical markers, which have differential diagnostic values in several forms of lymphoid tumors of B cell origin, will be important for the choice of treatment methods and prognosis.
\end{abstract} lymphoma.

Keywords: immunophenotype, cytochemistry, hairy cell leukemia, hairy cell leukemia variant, splenic marginal zone

DOI: $10.21303 / 2504-5695.2020 .001429$

\section{Introduction}

Lymphoid malignancies encompass an extremely heterogeneous group of diseases, based on their histological forms, biological and molecular genetic features, sites of clinical presentation (nodal or extranodal), tumor behavior (localized or disseminated), and response to the treatment [1-4].

According to the new revision of the World Health Organization classification [5], the delineation of separate forms of non-Hodgkin's lymphomas (NHL) is based on clinical findings and the data of pathologic and immunohistochemical studies and molecular-genetic analysis. The lymphoid tumors of B cell origin account for more than $90 \%$ of NHL. The separate group of B-cell lymphoid malignancies comprises the disorders, originated from the mature B cells at the antigen-dependent stages of their differentiation. Such diseases are characterized by the pronounced splenomegaly and the presence of neoplastic lymphoid cells with hairy-like projections of the cytoplasm. This group comprises hairy cell leukemia (HCL), HCL variant (HCL-v), splenic marginal zone lymphoma (SMZL), splenic diffuse red pulp small B-cell lymphoma (RPBL) [6-9].

The aim of the study consists in clarifying the nature of neoplastic cells in the above-mentioned group of B-cell lymphoid malignancies, based on the comparative analysis of enzymocytochemical and immunophenotypical features. 
We believe that only precise diagnosis of these types of B cell lymphomas in accordance with up-to-date classifications may represent the basis for further molecular biological studies. The insight into the nature of these tumors should be advantageous for improving our ability to evaluate patients and select the appropriate therapy.

\section{Patients and Methods}

The samples of peripheral blood and the sternal puncture of the bone marrow of 113 patients with various types of B-cell lymphoproliferative diseases are presented (Table 1). The patients were under observation and treatment at the specialized oncohematological facilities of Kyiv city and hematological units of the regional hospitals in 16 regions of Ukraine. The precise diagnostic studies were provided at the Oncohematology Department of R. E. Kavetsky Institute of Experimental Pathology, Oncology and Radiobiology, NAS of Ukraine. The whole study covered the period from January 2016 to December 2019 and was performed within the framework of the research project "Diagnostic study of the malignant diseases of lymphoid tissue". The study was approved by the decision of the Institutional Ethics Committee (Protocol No. 4 of 17.11 2015. The informed consent was obtained from all the patients, included in the study. All the samples were analyzed in patients prior to the beginning of the treatment.

Table 1

Patients with different types of B-cell lymphoproliferative diseases included into the study

\begin{tabular}{cccc}
\hline Nosological form & Number of patients & Age of patients (years) & Male to female ratio \\
\hline HCL & 45 & $48 \pm 22$ & $1.8: 1$ \\
HCL-V & 5 & $73 \pm 10$ & $4: 1$ \\
SMZL & 60 & $72.5 \pm 12.5$ & $1.6: 1.4$ \\
RPBL & 3 & $65 \pm 7$ & $2: 1$
\end{tabular}

The smears were stained with MGG and studied under the light microscope with immersion $(1000 \mathrm{x})$. The intracellular localization and activity of the tartrate-resistant acid phosphatase (TRAP) (EC 3.1.2.1) was analyzed by the method of Goldberg and Barka [10]. The expression of the surface antigens of cells was studied with the aid of the panel of monoclonal antibodies (MAb) (Dako Cytomation, Denmark) using the alkaline phosphatase streptavidin-biotin technique (LSAB-AP) for visualization. The cells were also stained with fluorochrome-conjugated MAbs and analyzed in Beckman Coulter EPICS XL cytometer (USA).

\section{Results and Discussion}

Practically all patients had splenomegalia at presentation. In few patients, the hyperplasia of the peritoneal and mediastinal lymph nodes was detected. Sometimes, the lesions in the liver and bones were found. Peritoneal or pleural exudates seem to be rare findings. According to the results of the complete blood test, most patients had anemia $(\mathrm{Hb}<100 \mathrm{~g} / \mathrm{l})$, thrombocytopenia $\left(<100 \cdot 10^{9} / 1\right)$ and leucopenia $\left(0.7-1.5 \cdot 10^{9} / 1\right)$. The fraction of villous lymphocytes in leukogram amounted to $5-10 \%$.

In HCL patients, the cell composition of myelogram varied. Sometimes, the extensive hypoplasia with single substrate-specific cells is evident. In other cases, lymphoid cells with characteristic cytomorphological features were predominant.

The principal enzymocytochemic diagnostic criterion of HCL is the presence of high acid phosphatase activity, revealed as the intensive staining in cytoplasm of the villous cells (Fig. 1). The addition of $0.05 \mathrm{M}$ sodium-potassium tartrate in staining mixture did not prevent enzymocytochemic detection of acid phosphatase. The presence of TRAP (isoenzyme 5) is very reliable feature, allowing for differentiate between HCL and other lymphoproliferative diseases.

The neoplastic cells in the bone marrow and peripheral blood of HCL patients had the immunophenotype of the relatively mature B cells. Their surface membranes express antigens, associated with B cells, such as CD19, CD20, CD22, CD79a (the two latter expressed most markedly). 
IgM was expressed moderately or strongly. Usually, CD5, CD10, CD23 and CD43 were not detected. We detected the expression of CD25 (alpha-chain of IL-2 receptor), representing the marker of B- and T-cell activation. The expression of CD1c was more pronounced than in other lymphoproliferative diseases. In contrast to B-CLL and a few cases of follicular lymphoma and mantle cell lymphoma, the neoplastic cells in HCL expressed CD103. In addition, CD200 expression was detected. In half of the cases, the weak expression of D1 cyclin was evident.

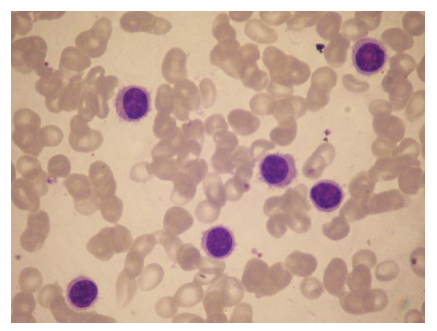

$a$

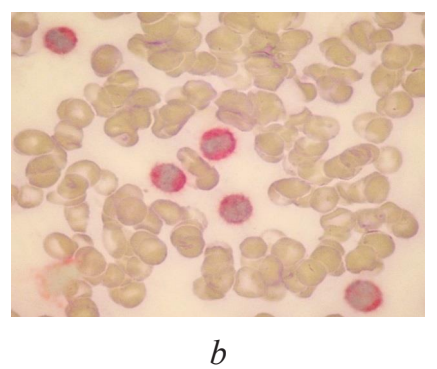

Fig. 1. Bone marrow cells in HCL: $a-$ MGG staining; $b-$ TRAP reaction, $\times 1000$

HCL-v differs from HCL by specific histological and immunophenotypical features as well as by the sensitivity to chemotherapy. In our HCL-v patients, splenomegalia was evident while lymphadenopathy was not detected. Anemia and thrombocytopenia were present in all patients. Leukocyte count in peripheral blood was within the range of 30-50 $10^{9} / 1$. In smears from the bone marrow and peripheral blood, the lymphoid cells with villous cytoplasm were noticeable.

The acid phosphatase reaction in cell cytoplasm has a small granular pattern, from weak to moderate. When tartrate was added to the incubation mixture, in two cases, acid phosphatase was still detected in a fraction of the cells, but the reaction intensity was weak. Nevertheless, in all other HCL-v patients, tartrate inhibited this reaction completely. B cell antigens, such as CD19 and CD20, were expressed on the surface of the cells in bone marrow and peripheral blood specimens. In addition, the weak expression of CD22, CD103 and CD11c was noticeable. The reaction with Mabs against CD25 and cyclin D1 was negative.

SMZL was earlier known as spleen lymphoma with circulating villous lymphocytesn and diagnosed as one of the cytological variants of B cell chronic lymphocytic leukemia (B-CLL). Most SMZL patients (49) in our study were presented with splenomegaly with the concomitant anemia and autoimmune thrombocytopenia. In 30 patients, lymph nodes, adjoining splenic hilum, were involved in the pathological process as well as the liver and the bone marrow. The peripheral lymph nodes were not affected. The leukocyte count in the peripheral blood was usually within the normal range or slightly elevated. Only in 12 patients (20\%), the leukocyte count exceeded $25 \cdot 10^{9} / 1$. The count of the villous lymphocyte in peripheral blood varied. The lymphocytes in peripheral blood were of small to medium size; sometimes their size was superior to that in B-CLL lymphocytes (Fig. 2).

The nuclei were rounded. The size of cytoplasm varied. In most cases, cytoplasm was stained light blue, with its area being narrower than in HCL, resulting in the increased nuclear-to-cytoplasm ratio. The fraction of lymphocytes had short few projections in cytoplasm.

In SMZL cells, the activity of the acid phosphatase was detected as weak to moderate staining of cytoplasm. In most patients, tartrate inhibited completely the acid phosphatase reaction in a 
majority of cells in bone marrow and peripheral blood smears. However, in $20 \%$ of the cases, tartrate did not inhibit the acid phosphatase reaction in 10-15\% of cells. Immunophenotyping finding proved that SMZL cells represented relatively mature B cells. The expression of CD19, CD20 and CD 22 was detected on the surface of the neoplastic cells. In half of the cases, CD11c expression was detected. In 6 patients $(10 \%)$, the weak expression of CD25 or CD5 was evident. CD103 and cyclin D1 were not expressed.

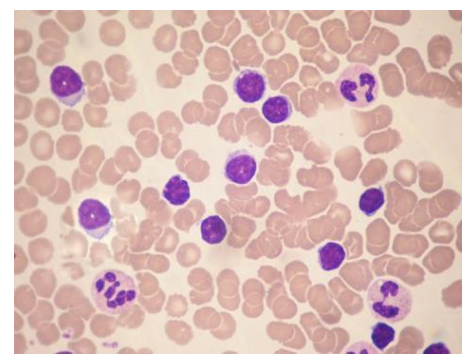

Fig. 2. B cells in peripheral blood in SMZL (leukemic phase); MGG, $\times 1000$

RPBL cases account for less than $1 \%$ of all NHL. The pathological process in RPBL involves not only the spleen but also the sinuses of the bone marrow. We have analyzed three RPBL cases (the age of the patients was 58, 64 and 72). The clinical and hematological features comprised the pronounced spenomegaly, leucopenia and thrombocytopenia. In bone marrow and peripheral blood smears, the rounded cells of small to moderate size were detected with the loose chromatin structure, narrow cytoplasm with features of basophilia. The cytoplasmic projections were somehow wider and longer than in cells of HCL and SMZL patients (Fig. 3).

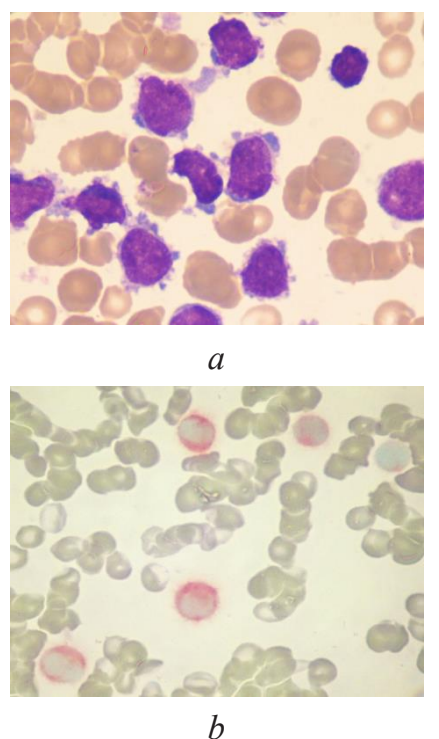

Fig. 3. Bone marrow cells in RPBL: $a-\mathrm{MGG} ; b-$ weak activity of TRAP, $\times 1000$

The activity of the acid phosphatase in the neoplastic cells upon cytochemical detection ranged from weak to moderate. In two patients, the reaction was completely inhibited by tartrate ions. In one patient, weak staining was detectable, following inhibition. The cells in the bone marrow and peripheral blood were characterized by the typical immunophenotype: $\mathrm{CD} 19^{+} \mathrm{CD} 20^{+} \mathrm{CD} 22^{+}$(bright) $\mathrm{CD} 25^{-} \mathrm{CD} 103^{-} \mathrm{CD} 11 \mathrm{c}^{-} \mathrm{CD} 5^{-}$cyclin $\mathrm{D}^{-}$. In one patient, $\mathrm{CD} 103$ and CD11c expression was slightly positive.

The study is limited only by the spectrum of monoclonal antibodies, used for the detection of the different markers of B cells. 
The prospects for further diagnostic immunocytochemical studies relate to the wide-scale application of the novel monoclonal antibodies against the lymphoid stem cells for detection of the potential markers of B cells at the different stages of antigen-dependent differentiation.

\section{Conclusions}

1. Our study revealed cytochemical and immunophenotypical features of villous cells, found in the bone marrow and peripheral blood of patients with HCL, HCL-v, SMZL, and RPBL. The data obtained confirm the justification for delineating the above lymphoid neoplasms as the separate nosological forms in the revised WHO classification (2016).

2. The marker immunocytochemical features of neoplastic cells, presented in our study, may be useful as the additional features for differential diagnosis of these forms of tumors upon histological and immunohistochemical study of the specimens of spleen, resected surgically in such patients.

3. The identification of small number of neoplastic cells in blood and bone marrow in the leukemic phase of B cell lymphoma proved to be possible using the panel of specifically selected monoclonal antibodies.

\section{Conflicts of interest}

This work does not have potential conflicts of interest.

\section{References}

[1] Feller, A. C., Diebold, J. (2004). Histopathology of Nodal and Extranodal Non-Hodgkin's Lymphomas. Springer. doi: https:// doi.org/10.1007/978-3-642-18653-0

[2] Jaffe, E. S., Harris, N. L., Stein, H., Isaacson, P. G. (2008). Classification of lymphoid neoplasms: the microscope as a tool for disease discovery. Blood, 112 (12), 4384-4399. doi: https://doi.org/10.1182/blood-2008-07-077982

[3] Swerdlow, S. H. (2008). WHO Classification of Tumors of Haematopoietic and Lymphoid Tissues. Lyon: IARC Press.

[4] Bain, B. J. (2017). Leukaemia diagnosis. John Wiley \& Sons. doi: https://doi.org/10.1002/9781119210511

[5] Swerdlow, S. H., Campo, E., Pileri, S. A., Harris, N. L., Stein, H., Siebert, R. et. al. (2016). The 2016 revision of the World Health Organization classification of lymphoid neoplasms. Blood, 127 (20), 2375-2390. doi: https://doi.org/10.1182/blood2016-01-643569

[6] Canellos, G. P., Lister, T. A., Young, B. (Eds.) (2006). The Lymphomas. Elsevier, 600. doi: https://doi.org/10.1016/b978-0-72160081-9.x5001-8

[7] Isaacson, P., Matutes, E., Burke, M., Catovsky, D. (1994). The histopathology of splenic lymphoma with villous lymphocytes. Blood, 84 (11), 3828-3834. doi: https://doi.org/10.1182/blood.v84.11.3828.bloodjournal84113828

[8] Sharpe, R. W., Bethel, K. J. (2006). Hairy Cell Leukemia: Diagnostic Pathology. Hematology/Oncology Clinics of North America, 20 (5), 1023-1049. doi: https://doi.org/10.1016/j.hoc.2006.06.010

[9] Matutes, E., Oscier, D., Montalban, C., Berger, F., Callet-Bauchu, E., Dogan, A. et. al. (2007). Splenic marginal zone lymphoma proposals for a revision of diagnostic, staging and therapeutic criteria. Leukemia, 22 (3), 487-495. doi: https://doi.org/ 10.1038/sj.leu.2405068

[10] Gluzman, D. F., Sklyarenko, L. M., Nadgornaya, V. A., Kryachok, I. A. (2003). Diagnosticheskaya immunotsitohimiya opuholey. Kyiv: Morion, 156. 